\title{
Role of Fractional Powers in maneuvering the Fractional Lower-Order Auto-Covariance of skewed alpha-stable signals in Gaussian Noise Environment
}

\author{
Areeb AHMED ${ }^{1}$ \\ Department of Electrical Engineering \\ ${ }^{1}$ Mohammad Ali Jinnah University \\ Karachi, Pakistan \\ areebahmed@iyte.com.tr
}

\author{
Ferit Acar SAVACI ${ }^{2}$, Muath WAHDAN ${ }^{2}$ \\ Department of Electrical and \\ Electronic Engineering \\ ${ }^{2}$ Izmir Institute of Technology \\ Izmir, Turkey \\ acarsavaci@iyte.com.tr,muathwahdan@iyte.edu.tr
}

\author{
Husam OTHMAN ${ }^{3}$ \\ Department of Electrical and \\ Electronic Engineering \\ ${ }^{3}$ Anadolu University \\ Eskisehir, Turkey \\ hzmo@anadolu.edu.tr
}

\begin{abstract}
Since, alpha-stable noise signals similarity can only be gauged by Fractional Lower-Order Auto-Covariance (FLOAC), therefore, the role of impulsiveness and skewness parameters, in generation and detection of the skewed alphastable (SkaS) noise signals, has been analyzed several times in the past. However, in this paper, a thorough analysis on the role of fractional powers in changing the FLOAC of SkaS noise signals has been carried out. The two associated fractional powers of FLOAC has been maneuvered in three possible ways to observe the probable trend of SkaS noise signals in the presence and absence of Gaussian noise. According to the observed results, the fractional powers largely and solely affect the FLOAC when they are manipulated collaboratively or even individually where the analyzed results can be handy in enhancing many SkoS noise signal processing techniques, especially, in the detection of SkaS noise carrier signals in Random Communication Systems.
\end{abstract}

Keywords-Fractional lower-order auto-covariance; Alphastable noise; Random communication system

\section{INTRODUCTION}

The concept of Fractional Lower-Order AutoCovariance (FLOAC) has been exploited in the past to enhance the efficiency of alpha-stable ( $\alpha$-stable) noise signal detection. In conventional communications, $\alpha$-stable noise has been used to depict the effect of Rayleigh fading and acoustic channels before $[1,2]$. However, it has also been a key factor in producing a noise model for molecular communication systems [3]. Similarly, the effect of $\alpha$-stable noise on neural networks has also been analyzed [4]. Moreover, alpha-stable noise signals have also been used as carrier signal to establish covert communications in an unconventional way in [5] which was later named Random Communication Systems (RCS) [6].

The FLOAC has helped in establishing as well as optimizing synchronization method in RCSs [7, 8] which was established after analyzing the role of impulsive and skewness parameters in fluctuating the FLOAC [9]. However, no detailed study has been carried out yet to explore the role of fractional powers in maneuvering the FLOAC. Therefore, the result obtained in this paper would benefit different $\alpha$-stable noise signal detection schemes-based systems, especially RCSs. In the following Section-II and Section III, $\alpha$-stable noise distribution and FLOAC has been briefly explained, respectively. In Section IV, the observed results are presented where the paper has been concluded in Section- $\mathrm{V}$.

\section{AlPha-StABLE Distribution}

The samples of $\alpha$-stable noise can be produced via method given in [10] to generate the $\alpha$-stable distribution. The sequence of $\alpha$-stable noise, i.e. collection of consecutive $\alpha$-stable noise samples, is represented by $\mathrm{X} \sim \mathrm{S}_{\alpha}(\beta, \gamma, \mu)$ where $\alpha$ has been defined as the impulsiveness parameter in the range $(0<\alpha \leq$ 2), $\beta$ has been defined as the skewness parameter defined in the range $\beta(-1 \leq \beta \leq 1), \gamma$ has been defined as the dispersion parameter in the range $(\gamma \geq 0)$ and $\mu$ has been defined as the location parameter which is $\mu \in R$ in [11]. The characteristic function of $\mathrm{X} \sim \mathrm{S}_{\alpha}(\beta, \gamma, \mu)$ is expressed in [11] as

$$
\begin{aligned}
& \phi(\theta)= \\
& \left\{\begin{array}{c}
\exp \left\{j \mu \theta-\gamma^{\alpha}|\theta|^{\alpha}\left(1-j \beta \operatorname{sign}(\theta) \tan \left(\frac{\alpha \pi}{2}\right)\right)\right\} \text { if } \alpha \neq 1 \\
\exp \left\{j \mu \theta-\gamma|\theta|\left(1+j \beta \frac{2}{\pi} \operatorname{sign}(\theta) \ln \left(\frac{\alpha \pi}{2}\right)\right)\right\} \text { if } \alpha=1
\end{array}\right.
\end{aligned}
$$

Note: $\mathrm{X}_{\mathrm{G}} \sim \mathrm{S}_{\alpha=2}\left(\beta=0, \gamma_{G}, \mu_{G}\right), \mathrm{X}_{\mathrm{C}} \sim \mathrm{S}_{\alpha=1}\left(\beta=0, \gamma_{C}, \mu_{C}\right), \mathrm{X}_{\mathrm{L}} \sim$ $\mathrm{S}_{\alpha=0.5}\left(\beta=1, \gamma_{L}, \mu_{L}\right)$ generates Gaussian, Cauchy and Levy distributed samples, respectively, as these are special cases of $\alpha$-stable distributions.

\section{FRACTIONAL LOWER-ORDER AUTO-COVARIANCE}

All conventional time delay estimation methods, i.e. Covariance and Correlation, cannot be applied for signal processing of $\alpha$-stable noise signals for $\alpha<2$ due to the nonpresence of moments greater than second-order for $\alpha$-stable distribution. Therefore, FLOAC method has always been exploited to check resemblance among $\alpha$-stable noise signals [9, $12]$.

If the FLOAC of $\alpha$-stable noise signal $\mathrm{X} \sim \mathrm{S}_{\alpha}(\beta, \gamma, \mu)$ is defined as

$$
\boldsymbol{R}_{\boldsymbol{d}}[\boldsymbol{k}] \triangleq \boldsymbol{E}\left\{(x[i])^{a} \cdot(x[i+k])^{b}\right\}
$$




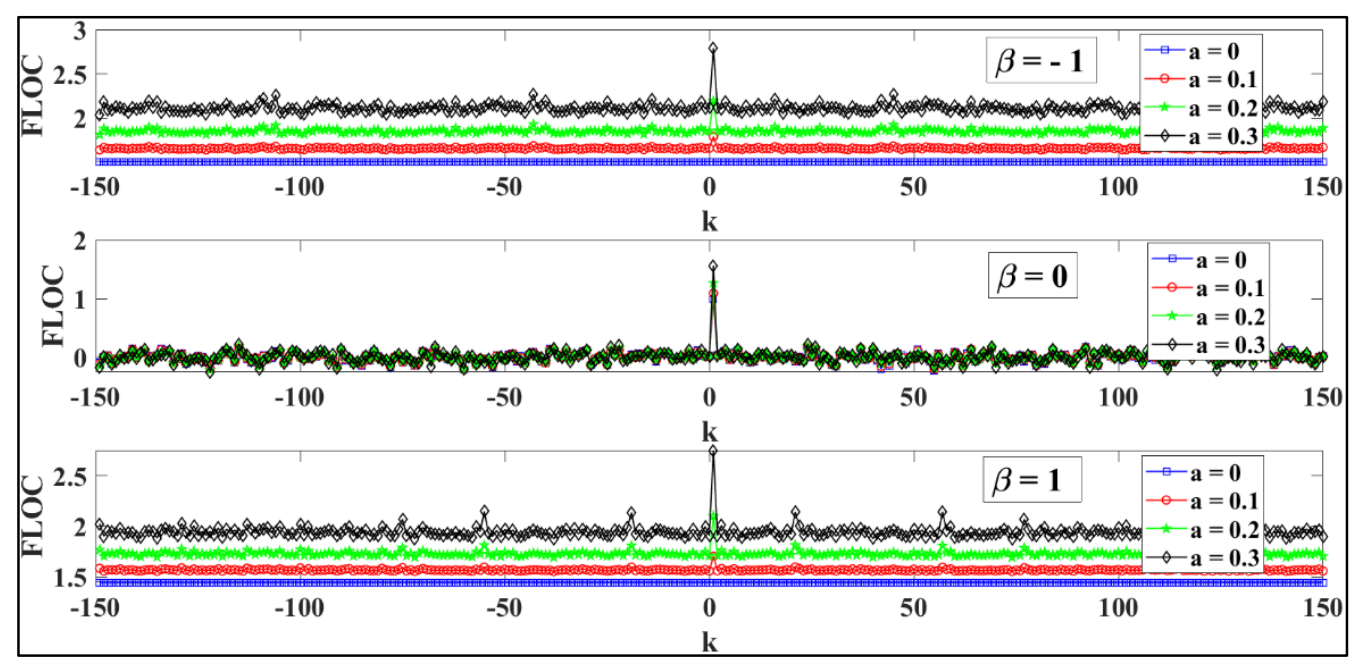

Fig. 1: $\boldsymbol{R}_{\boldsymbol{d}}[\boldsymbol{k}]$ for $\mathrm{S}{ }_{\alpha=0.75}(\beta=-1,0,1, \mu=0), \boldsymbol{M S} \boldsymbol{N} \boldsymbol{R}_{\boldsymbol{d} \boldsymbol{B}}=-4 \mathrm{~dB}$ with various 'a'

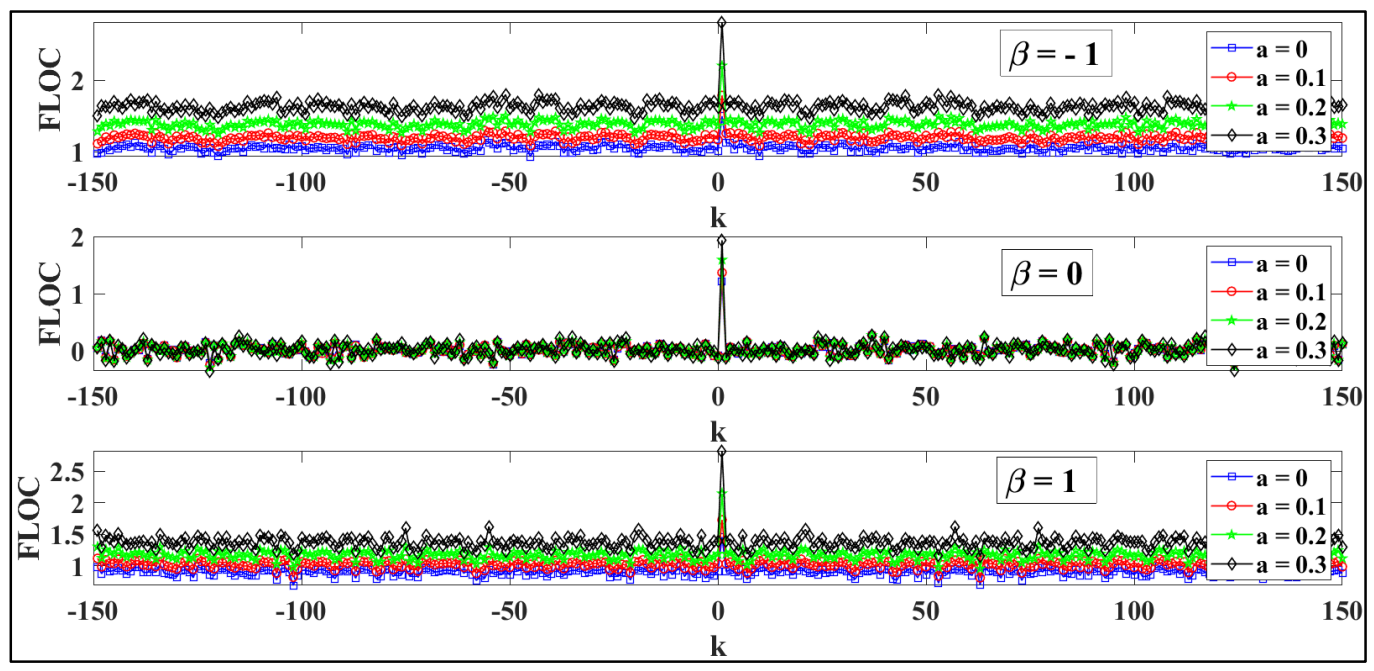

Fig. 2: $\boldsymbol{R G}_{\boldsymbol{d}}[\boldsymbol{k}]$ for $\mathrm{S} \alpha=0.75(\beta=-1,0,1, \mu=0), \boldsymbol{M} \boldsymbol{S} \boldsymbol{N} \boldsymbol{R}_{\boldsymbol{d B}}=-4 \mathrm{~dB}$ with various ' $\mathrm{a}$ '

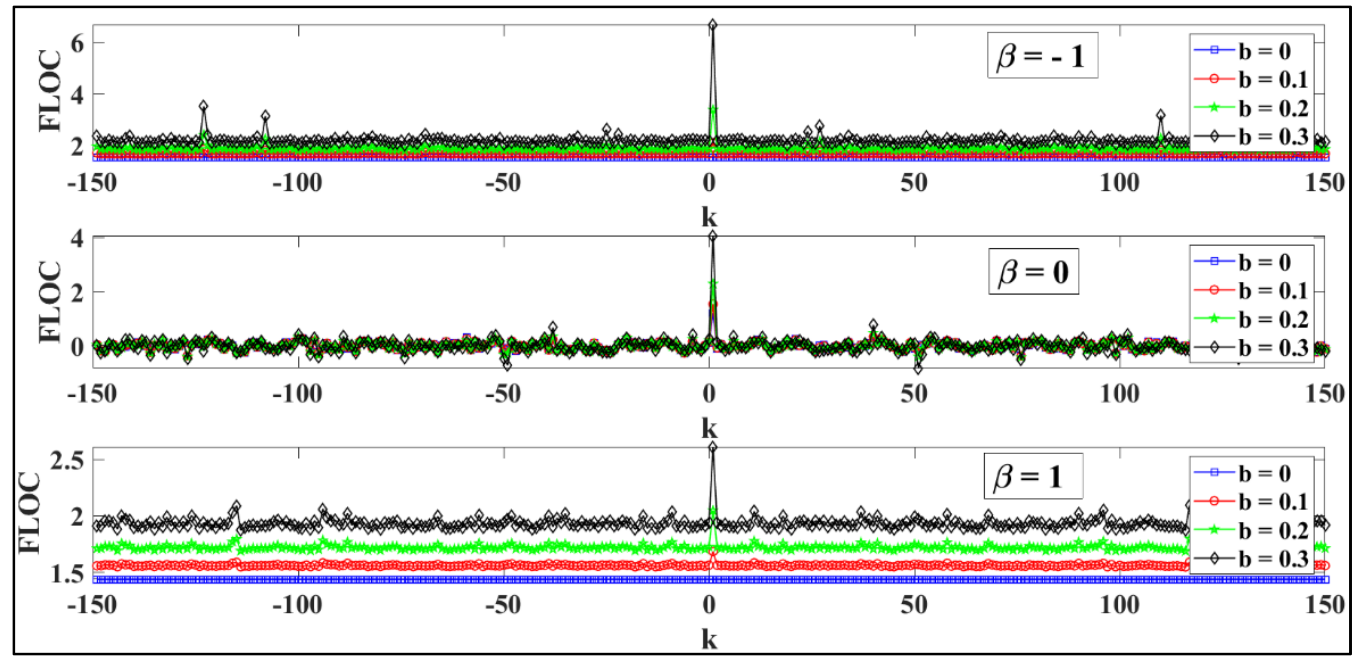

Fig. 3: $\boldsymbol{R}_{\boldsymbol{d}}[\boldsymbol{k}]$ for $\mathrm{S}_{\alpha=0.75}(\beta=-1,0,1, \mu=0), \boldsymbol{M} \boldsymbol{S} \boldsymbol{N} \boldsymbol{R}_{\boldsymbol{d B}}=-4 \mathrm{~dB}$ with various 'b' 


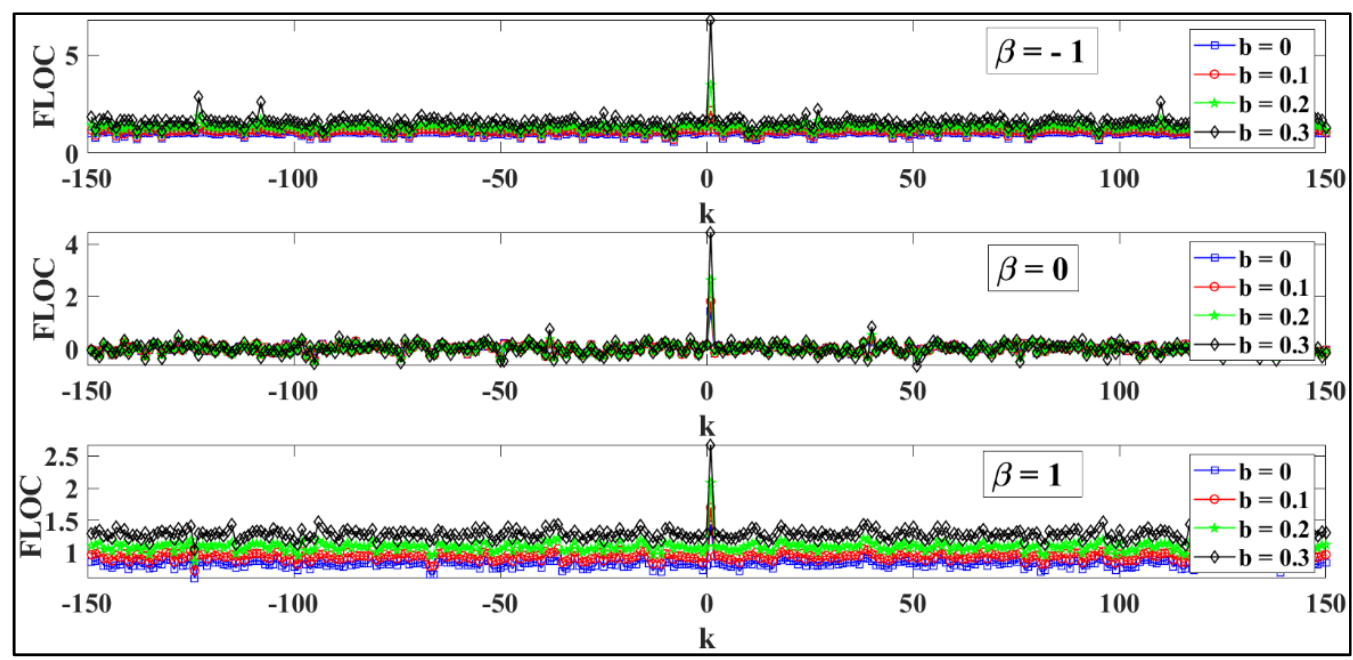

Fig. 4: $\boldsymbol{R}_{\boldsymbol{d}}[\boldsymbol{k}]$ for $\mathrm{S}_{\alpha=0.75}(\beta=-1,0,1, \mu=0), \boldsymbol{M S N} \boldsymbol{R}_{\boldsymbol{d B}}=-4 \mathrm{~dB}$ with various 'b'

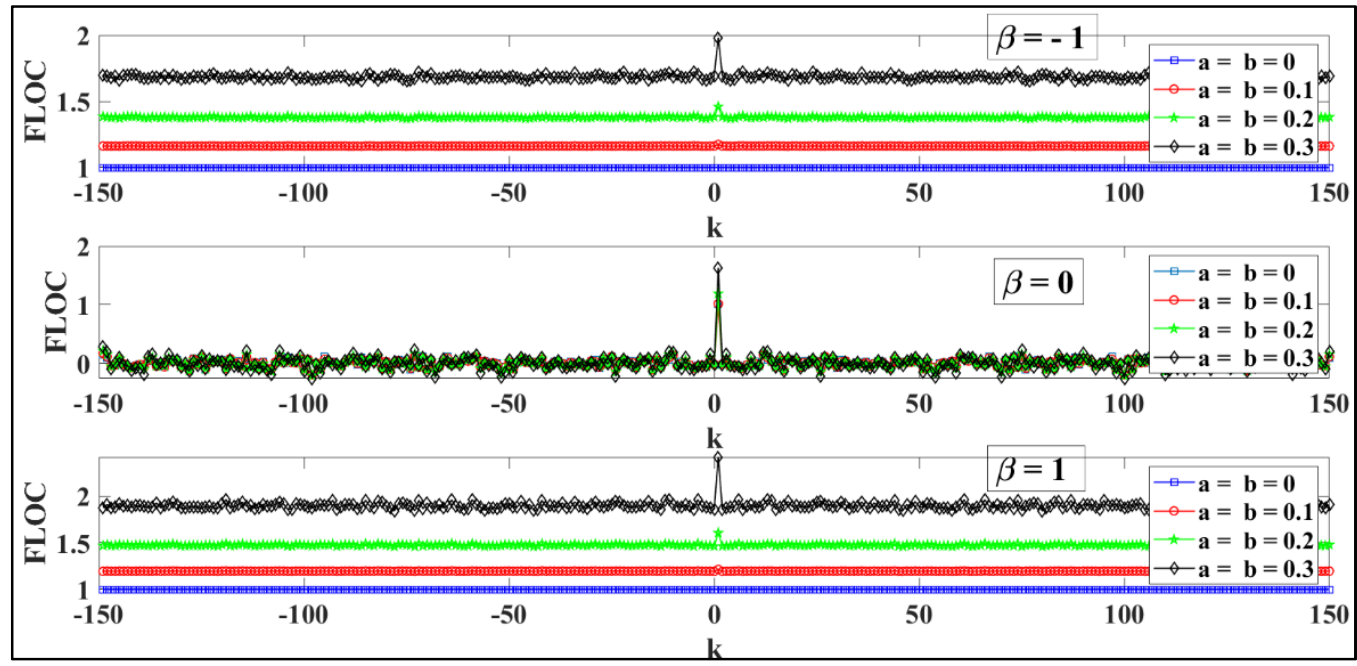

Fig. 5: $\boldsymbol{R}_{\boldsymbol{d}}[\boldsymbol{k}]$ for $\mathrm{S}{ }_{\alpha=0.75}(\beta=-1,0,1, \mu=0), \boldsymbol{M S N} \boldsymbol{R}_{\boldsymbol{d B}}=-4 \mathrm{~dB}$ with various ' $\mathrm{a}=\mathrm{b}$ '

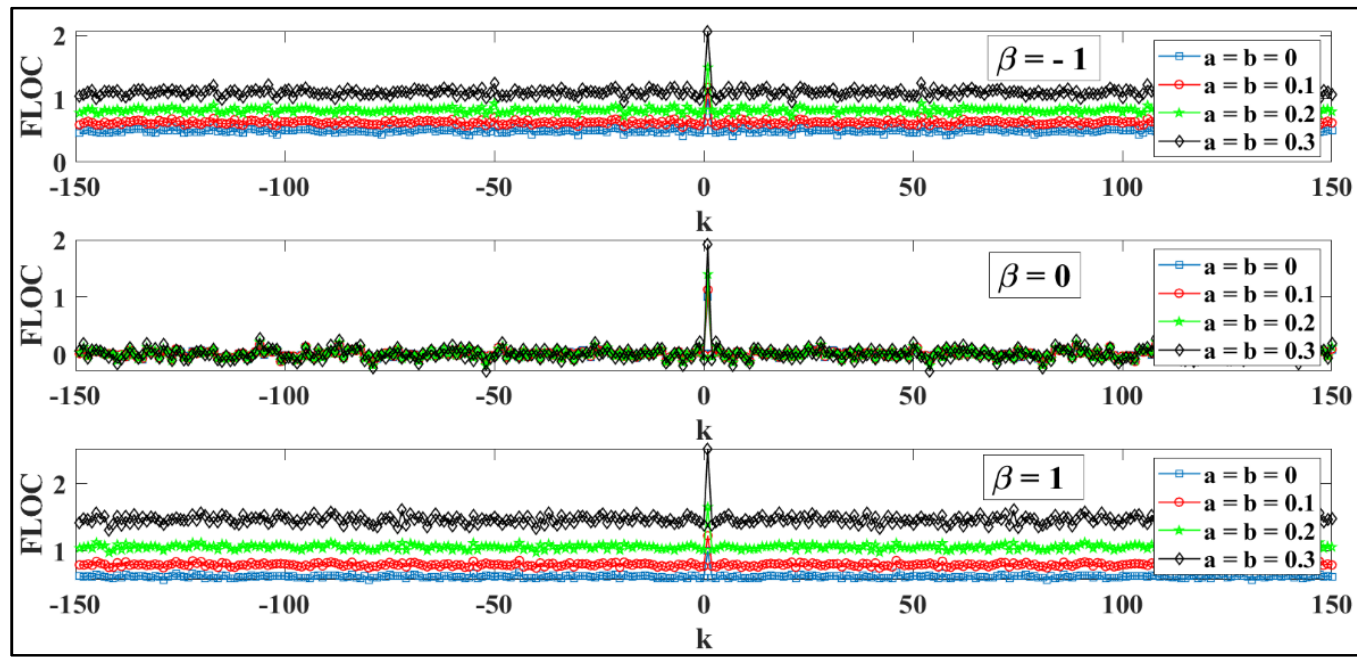

Fig. 6: $\boldsymbol{R}_{\boldsymbol{d}}[\boldsymbol{k}]$ for $\mathrm{S}_{\alpha=0.75}(\beta=-1,0,1, \mu=0), \boldsymbol{M S N} \boldsymbol{R}_{\boldsymbol{d B}}=-4 \mathrm{~dB}$ with various ' $\mathrm{a}=\mathrm{b}$ ' 
then the FLOAC, denoted by $R_{d}[k]$, of $\mathrm{X}$, i.e. $\mathrm{X}=$ $\{x[1], \ldots, x[n] ; n=1,2, \ldots, N\}$, is estimated in [12] as

$$
\boldsymbol{R}_{\boldsymbol{d}}[\boldsymbol{k}]=\frac{\sum_{n=N_{1}+1}^{N_{2}}|x[n]|^{a} \cdot|x[n+k]|^{b} \cdot \operatorname{sign}(x[n] \cdot x[n+k])}{N_{2}-N_{1}}
$$

where $N_{1}=\max (0,-k), N_{2}=\min (N-k, N), k=\left(\frac{-N}{2}, \frac{N}{2}\right)$. Similarly, the noisy FLOAC, $R G_{d}[k]$, of $\alpha$-stable noise signal $\mathrm{X} \sim \mathrm{S}_{\alpha}(\beta, \gamma, \mu)$ added with $\mathrm{X}_{\mathrm{G}} \sim \mathrm{S}_{\alpha=2}\left(\beta=0, \gamma_{G}, \mu_{G}\right)$, i.e. $\mathrm{X}+$ $\mathrm{X}_{\mathrm{G}}$, has been computed as

$$
\begin{aligned}
& \boldsymbol{R} \boldsymbol{G}_{\boldsymbol{d}}[\boldsymbol{k}]= \\
& \frac{\sum_{n=N_{1}+1}^{N_{2}}\left|x+x_{G}[n]\right|^{a} \cdot\left|x+x_{G}[n+k]\right|^{b} \cdot \operatorname{sign}\left(x+x_{G}[n] \cdot x+x_{G}[n+k]\right)}{N_{2}-N_{1}}
\end{aligned}
$$

However, $R_{d}[k]$ and $R G_{d}[k]$ has always been exploited for fixed value of fractional powers which is usually fixed as $a=$ $b=\frac{\alpha}{2}[5,7-9,13]$ and the complete effects of maneuvering the fractional powers on $R_{d}[k]$ has not been analyzed yet.

\section{ANALYSIS OF FRACTIONAL POWERS}

Therefore, in this section of the paper, the FLOACs, i.e. $R_{d}[k]$ and $R G_{d}[k]$ in (3) and (4), respectively, has been computed by maneuvering 'a' and ' $b$ ' while keeping $\alpha=0.75$ and $\mu=0$ intact. The FLOAC, i.e. $R_{d}[k]$ in (3), has been analyzed for three possible values of skewness parameter $\beta$, i.e. $\beta=-1,0,1$, to completely analyzed the behavior of FLOAC of skewed $\alpha$-stable signals when the ' $a$ ' and ' $b$ ' are varied individually and collaboratively in three possible ways. Firstly, in Fig 1 and Fig. 2, only the fractional power ' $a$ ' has been varied, i.e. $\mathrm{a}=0,0.1,0.2,0.3$, while keeping the ' $\mathrm{b}$ ' constant, i.e. $b=0.15$. Secondly, in Fig 3 and Fig. 3, only the fractional power ' $b$ ' has been varied, i.e. $b=0,0.1,0.2,0.3$, while keeping the ' $a$ ' constant, i.e. $a=0.15$. Finally, in Fig 5 and Fig. 6 , both the fractional power ' $a$ ' and ' $b$ ' has been varied, i.e. $a=b=0$, $0.1,0.2,0.3$. The simulations shown in Fig. $1-6$ have been made for $N=300$ and $M S N R=-4 \mathrm{~dB}$ where the MSNR is defined in [12] as $M S N R_{d B}=10 \log \frac{\gamma}{\gamma_{G}}$ where $\gamma_{G}$ is the dispersion parameter the gaussian noise $\mathrm{G}$ and $\gamma$ is the dispersion parameter of the $\alpha$-stable noise signal X.

The FLOAC, i.e. $R_{d}[k]$ in (3), shows an interesting behavior as ' $a$ ' and ' $b$ ' are varied in the three different ways. In the first case, the FLOACs $R_{d}[k]$ and $R G_{d}[k]$ has been computed in Fig. 1 and Fig.2, respectively, when only 'a' is varied where it has been seen that $R_{d}[k]$ and $R G_{d}[k]$ increases when 'a' increases up to the maximum limit of a, i.e. $\mathrm{a} \leq \frac{\alpha}{2}$. In the second case, the FLOACs $R_{d}[k]$ and $R G_{d}[k]$ has been computed in Fig. 3 and Fig. 4, respectively, when only 'b' is varied where it has been seen again that $R_{d}[k]$ and $R G_{d}[k]$ increases when 'b' increases up to the maximum limit of b, i.e. $b \leq \frac{\alpha}{2}$. In the third case, the FLOACs $R_{d}[k]$ and $R G_{d}[k]$ has been computed in
Fig. 5 and Fig. 6, respectively, when both 'a' and 'b' are varied where it has been seen again that $R_{d}[k]$ and $R G_{d}[k]$ increases when ' $a$ ' and ' $b$ ' increases up to the maximum limit of $a$ and $b$, i.e. $\mathrm{a}=\mathrm{b} \leq \frac{\alpha}{2}$. Moreover, among the three used values of $\beta$, i.e. $\beta=-1,0,1$, in every Fig. 1-6, the maximum value of $R_{d}[0]$ and $R G_{d}[0]$ has always been observed at $\beta=-1$ and $\beta=1$ which shows that utilizing skewed $\alpha$-stable signals are beneficial when high values of FLOAC is required and vice versa.

\section{CONCLUSION}

In this paper, Fractional lower-order auto-covariance has been analyzed while maneuvering the fractional powers in three different ways. It has been found that the maximum value of FLOAC can be obtained for skewed $\alpha$-stable signals if higher values of fractional powers are utilized and vice versa. Similarly, $\alpha$-stable signals skewed to extreme right or left produces maximum FLOAC values. The generalized results would help different $\alpha$-stable noise signal detection schemes-based systems.

\section{REFERENCES}

[1] A. Rajan and C. Tepedelenlioglu, "Diversity combining over Rayleigh fading channels with symmetric alpha-stable noise," IEEE Transactions on Wireless Communications, vol. 9, no. 9, pp. 2968-2976, 2010.

[2] H. He, J. Lu, J. Chen, X. Qiu and J. Benesty, "Robust blind identification of room acoustic channels in symmetric alpha-stable distributed noise environments," The Journal of the Acoustical Society of America, vol. 136, no. 2, pp. 693-704, 2014.

[3] N. Farsad, W. Guo and C.B. Chae, "Stable distributions as noise models for molecular communication," in Global Communications Conference (GLOBECOM), 2015, pp. 1-6.

[4] B. Kosko and S. Mitaim, "Stochastic resonance in noisy threshold neurons," Neural networks, vol. 16, no. 5, pp. 755-761, 2010.

[5] M.E. Cek, "Covert communication using skewed $\alpha$-stable distributions," IET Electronics Letters, vol. 51, no. 1, pp. 116-118, 2015.

[6] A. Ahmed and F.A Savaci, "Random Communication System Based on Skewed Alpha-Stable Levy Noise Shift Keying," Fluc. and Noise Letters , vol. 16, no. 3, 1750024, 2017.

[7] A. Ahmed and F.A Savaci, "Synchronization of alpha-stable Levy noise based random communication system," IET comunications, vol. 12, no. 3, pp.276-282. 2017.

[8] A. Ahmed and F.A Savaci, "On optimizing fractional lower order covariance based synchronization method for random communication systems," in 26th Signal Processing and Communications Applications Conference (SIU), 2018, pp. 1-4..

[9] A. Ahmed and F.A Savaci, "Structure and Performance Evaluation of Fractional Lower-Order Covariance Method in Alpha-Stable Noise Environments," Recent Advances in Electrical \& Electronic Engineering (Formerly Recent Patents on Electrical \& Electronic Engineering), vol. 12, no.1, pp.40-44, 2019.

[10] A. Janicki and A. Weron, Simulation and chaotic behavior of a-stable stochastic processes, C.\&Hall/CRC, 1994.

[11] G. Samorodnitsky and M. Taqqu, Stable non-gaussian random processes: stochastic models with infinite variance, C.\&Hall/CRC, 1994

[12] X. Ma and C.L. Nikias, "Joint estimation of time delay and frequency delay in impulsive noise using fractional lower order statistics," IEEE Transactions on Signal Processing, vol. 44, no.11, pp. 2669-2687, 1996.

[13] W. Liu, Y. Wang and T. Qiu, "Evoked potential latency delay Estimation by using covariation correlation approach," in The 2nd International Conference on Bioinformatics and Biomedical Engineering (ICBBE), 2008, pp. 652-655. 